\title{
Older age and high BMI are associated with increased risk of loss of kidney function
}

Several reports have suggested that the effects of obesity might differ between older and younger individuals, but the effect of age on the association of obesity with deterioration of kidney function was previously unknown. Now, research performed by Csaba Kovesdy and colleagues has shown a marked association between high BMI and loss of kidney function in patients aged $>40$ years.

The researchers analysed data collected during clinical practice from a cohort of $>3$ million US veterans with estimated glomerular filtration rates $>60 \mathrm{ml} /$ $\mathrm{min} / 1.73 \mathrm{~m}^{2}$. The large size of the study cohort enabled the participants, most of whom were male, to be categorized according to age in 10-year increments from $<40$ years to $\geq 80$ years. Kovesdy and colleagues found no relationship between $\mathrm{BMI}$ and kidney function in the youngest age group, but increasingly strong associations between BMI and loss of kidney function in the older age groups.
The best clinical outcomes were observed in patients with BMIs $>25 \mathrm{~kg} / \mathrm{m}^{2}$ but $<30 \mathrm{~kg} / \mathrm{m}^{2}$. The researchers also examined the relationship between BMI and allcause mortality and found U-shaped associations in all age groups.

"These observations have not been previously reported by others," says Kovesdy. "A potential explanation for our findings might be that a long time is needed for obesity to damage the kidneys (hence a more marked effect among

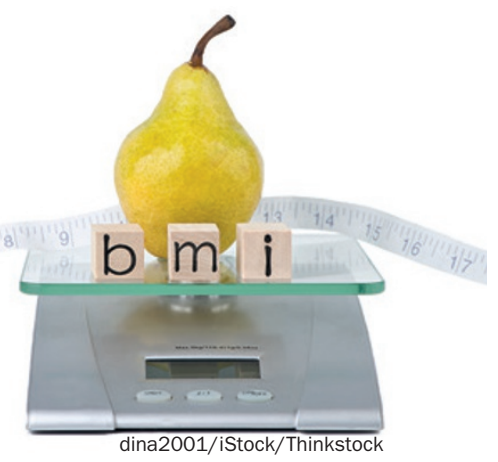

those with the longest exposures), or that the deleterious effects of advanced age and obesity conspire to cause enhanced damage to the kidneys of older individuals."

The researchers would like to see their findings replicated in other cohorts. Furthermore, they propose that clinical trials should be performed to determine the ideal BMI and at what age obesity control would be most effective as a renoprotective intervention. "Obesity has reached epidemic proportions and the world's population is ageing," explains Kovesdy. "Therefore, it is important that we continue to assess how obesity affects clinical outcomes among younger and older individuals".

Jessica K. Edwards

Original article Lu, J. L. et al. Association of age and BMI with kidney function and mortality: a cohort study. Lancet Diabetes Endocrinol. doi: 10.1016/S22138587(15)00128-X 